\title{
Evaporation of ethanol/water mixture droplets on micro-patterned PDMS surfaces
}

\author{
Ying-Song $\mathrm{Yu}^{\mathrm{a}, *}$, Xianfu Huang ${ }^{\mathrm{b}}$, Li Sun ${ }^{\mathrm{a}}$, Jin-Zhi Zhou ${ }^{\mathrm{a}, *}$, An Zhou ${ }^{\mathrm{a}}$ \\ ${ }^{a}$ Department of Mechanics, School of Civil Engineering, Architecture and Environment, Hubei University of Technology, Wuhan 430068, China \\ ${ }^{\mathrm{b}}$ State Key Laboratory of Nonlinear Mechanics, Institute of Mechanics, Chinese Academy of Sciences, Beijing 100190, China
}

\section{A R T I C L E I N F O}

\section{Article history:}

Received 10 July 2019

Received in revised form 27 August 2019

Accepted 6 September 2019

\section{Keywords:}

Mixture droplet

PDMS

Evaporation

Wettability

Constant contact radius mode

\begin{abstract}
A B S T R A C T
We report evaporation of ethanol/water mixture droplets on both pillar-arrayed and micro-holed polydimethylsiloxane (PDMS) surfaces. Analysis of wettability shows that (i) when the pillar-to-pillar spacing is $5 \mu \mathrm{m}$, the pillar-arrayed PDMS surfaces are Cassie-Baxter-wetted; (ii) when the pillar-to-pillar spacing is 20 or $50 \mu \mathrm{m}$, part of each pillar-arrayed surface is Cassie-Baxter-wetted while the other part Wenzelwetted; (iii) all micro-holed PDMS surfaces are Cassie-Baxter-wetted; (iv) the advancing and receding contact angles increase with the increase of the pillar-to-pillar spacing, while they decrease with the increase of the hole-to-hole spacing. The investigation on evaporation of mixture droplets demonstrates that (i) evaporation on pillar-arrayed PDMS first proceeds with constant contact radius mode (CCR) due to the strong adhesion between the surface and the droplet; (ii) on micro-holed surfaces CCR stage is also observed for the surface with the hole-to-hole spacing of $5 \mu \mathrm{m}$, while it is not found at high ethanol concentration for surfaces with the hole-to-hole spacings of $20 \mu \mathrm{m}$ and $50 \mu \mathrm{m}$, which is attributed to the weak adhesion between the micro-holed surfaces and the droplet. Finally, the evolution of droplet volume and its two-third power are discussed.
\end{abstract}

(c) 2019 Elsevier Ltd. All rights reserved.

\section{Introduction}

Drop evaporation has many applications in fields such as microfluidics, lab-on-a-chip, ink-jet printing, combustion and pesticide spraying [1-7]. In 1977, Picknett and Bexon [8] pioneeringly defined three evaporation modes, viz., constant contact radius mode (CCR mode), constant contact angle mode (CCA mode) and mixed mode. At first, the contact line is pinned due to contact angle hysteresis and the the contact angle decreases until it reaches the receding angle. Then the contact line begins to recede and the contact angle keeps nearly unchanged. Finally, both the contact radius and the contact angle decrease with time. These three modes are found in evaporation of water droplets on both hydrophilic and hydrophobic surfaces [9-11].

During the latest decades, evaporation of mixture droplet has aroused wide interest. The evaporation of sessile ethanol/water [12-17] or 1-propanol-water [18] or water-ethylene glycol [19] or methanol-water [20] mixture droplet on planar surface has been extensively studied. Organic solvents such as ethanol and glycerol have strong volatility. When a mixture droplet containing water and one of these solvents evaporates on a solid surface, there is a

\footnotetext{
* Corresponding authors.

E-mail addresses: yuys@hbut.edu.cn (Y.-S. Yu), zhoujz@hbut.edu.cn (J.-Z. Zhou).
}

strong volatilization, which may induce a concentration gradient inside the droplet and result in a Marangoni flow inside the droplet. Moreover, such a flow will change the shape of the droplet [21]. Using the particle image velocity, Christy et al. [22] studied the flow field near the solid-liquid surface of an evaporating water-ethanol droplet and observed three different stages, viz., multiple vortices, spike-like outward flow stage and outward flow similar to that found in pure water droplet.

Surfaces with microstructures such as micro-pillars and micropores have some applications in fabrication of superhydrophobic or superhydrophilic surfaces and oil/water inside the rocks [23-29]. Yuan et al. [24,30-31] elegantly studied the wettability of patterned surfaces. Combining experiments with molecular dynamics simulations, they investigated dynamic wetting of a droplet on lyophilic pillar-arrayed surfaces and found that $R$ scaled, respectively, with $t^{1 / 3}$ and $t^{1 / 7}$ for rough and smooth surfaces [30]. They [31] developed a theoretical model considering the coupling effects of viscous resistance and molecular friction to explain the dynamic spreading of a droplet on micropillar-arrayed surfaces. They also studied the comprehensive influence of surface topology, the intrinsic wettability and substrate elasticity on dynamic wetting on hydrophilic pillar-arrayed surfaces [24]. Recently, Chen et al. [32] fabricated the quasi-periodic surface structure to control 
the transition of droplets and for the first time found 5-fold symmetric shapes on seemingly random-like surface structures.

Evaporation of water droplet on patterned surfaces has also attracted wide interest. Anantharaju et al. [33] experimentally studied evaporation of sessile droplets on micro-patterned surfaces with square pillars or square holes and observed pinned triple line phase and moving triple line phase. They found that the dimensionless evaporation rate constant is higher in the moving triple line phase as compared with that in the pinned triple line phase, and the triple line topology has no influence on the evaporation rate constant. Evaporation of sessile water droplets on patterned PDMS surfaces was experimentally studied by Luo et al. [34], Zhang et al. [35] and Chuang et al. [36]. Luo et al. [34] found that the inclined angles of the PDMS microline sidewalls have great influence on the wettability of evaporating water droplet. Zhang et al. [35] experimentally studied evaporation of water droplets on PDMS spherical cap arrays and set up a model considering the pinning force of the contact line, which was used to clarify the observed different evaporation modes and the transition from pinned contact line to shrinking contact line. Chuang et al. [36] experimentally studied evaporation of sessile water droplets on regular pillar-like PDMS surfaces with different mixing ratio. They found that the receding angle becomes smaller with a softer surface. And the evaporation proceeds first with CCR mode, switches to CCA mode and finally shifts to the mixed mode. Evaporation of water droplets on superhydrophobic surfaces [11,37-39] has also been studied. Chen et al. [11] found that surface roughness has a remarkable influence on the transition from CCR to CCA and the critical base size at the transition from Cassie mode to Wenzel mode. Xu et al. [38] reported that CCR mode and CCA mode dominate the evaporation process and the duration of CCR stage becomes shorter with decreasing solid fraction. While, that of CCA stage becomes longer. Using molecular dynamic simulation, Wang and $\mathrm{Wu}[40]$ found that the localized liquid-solid interaction near the contact line greatly influences the evaporation mode of nanodrops on textured droplet, that is, CCR stage will be first observed in the case of a stronger interaction while CCA stage is observed first in the case of a weaker interaction. Furthermore, they studied the surface flexibility on evaporation of nano-drop on nano-pillared surfaces and concluded that the competition between pinning and depinning force is the cause of stick-slip motion of the contact line [41]. Recently, He et al. [42] studied evaporation of ethanol/water mixture droplet on chemical micropatterned surfaces with constant substrate surface temperature of $40^{\circ} \mathrm{C}$ and they found that CCR stage and moving contact line stage were both observed consequently. And they developed a local force model to elucidate the stepwise movement of the contact line. In 2009, Yu et al. [43] studied evaporation of ethanol/ water mixture droplets on pillar-arrayed PDMS surface and set up a theory based on Taylor's expansion to explain the spontaneous spreading during droplet evaporation.

In this work, we study the wettability of pillar-arrayed and micro-holed PDMS surfaces and evaporation of ethanol-water mixture droplets on these surfaces. Pillar-arrayed PDMS surfaces with the pillar-to-pillar spacing of $5 \mu \mathrm{m}$ are Cassie-Baxter-wetted and for pillar-arrayed surfaces with the pillar-to-pillar spacing of 20 or $50 \mu \mathrm{m}$, part of each surface is Cassie-Baxter-wetted and the other part Wenzel-wetted. All micro-holed PDMS surfaces are Cassie-wetted. The advancing and receding contact angles increase with the increase of the pillar-to-pillar spacing, while they decrease with the increase of the hole-to-hole spacing. CCR mode is found in most of the evaporation experiments of mixture droplets on micro-patterned PDMS surface and the duration of the CCR stage increases with the increase of the pillar-to-pillar spacing, while it decreases with the increase of the hole-to-hole spacing.
Evolution of droplet volume and its two-third power as well as evaporation rate are also discussed.

\section{Materials and method}

Silicon masters with micropillars or microholes were prepared by photolithography. They were first ultrasonically stirred successively in ethanol and acetone for about $10 \mathrm{~min}$ to ensure that the surfaces were clean. After about 5-minute oxygen plasma treatment (Harrick PDC-002 setup), the clean masters were further modified by a self-assembled chlorotrimethylsilane (Aladdin) monolayer to minimize its surface energy. PDMS (Sylgard 184, Dow corning, USA) with 10:1 mass ratio of base to curing agent was mixed well and vacuumed for about $30 \mathrm{~min}$ to extract all the visible bubbles. Then the PDMS was poured onto the masters and spin-coated successively at the rate of $400 \mathrm{rpm}$ for $20 \mathrm{sec}$ and $1000 \mathrm{rpm}$ for $30 \mathrm{sec}$, and then it was vacuumed for $30 \mathrm{~min}$ to extract the bubbles between PDMS and the masters. After 3-hour thermal curing at $90^{\circ} \mathrm{C}$, regular micro-patterned PDMS substrates were obtained by peeling off the PDMS molds from the patterned silicon masters and placed on clean cover glass. Pillar/hole diameter $d$ and pillar/hole height $h$ are both $20 \mu \mathrm{m}$. The pillar-to-pillar/hole-to-hole spacings $w$ are 5, 20 and $50 \mu \mathrm{m}$, respectively. The micro-patterned PDMS substrates were characterized using scanning electronic microscopy (SEM, FEI Quanta 200 FEG, Nederland), as illustrated in Fig. 1.

Ethanol/water mixture droplets with initial volume ranging from 1.90 to $3.05 \mu \mathrm{L}$ (the initial volume concentration of ethanol is $0 \%, 25 \%, 50 \%$ and $75 \%$, respectively) were deposited from the tip of the NE31-type needle on micro-patterned PDMS surfaces and observed using a droplet shape analyzer - DSA30 system (precision: $\pm 0.1^{\circ}$, Krüss, Germany) equipped with a high-resolution camera at $1 \mathrm{fps}$. The environmental temperature and relatively humidity of mixture droplets evaporating on pillar-arrayed PDMS surfaces were $26.5^{\circ} \mathrm{C} \pm 1^{\circ} \mathrm{C}$ and $52 \pm 2 \%$, respectively.

The environmental temperature and relatively humidity of mixture droplets evaporating on micro-holed PDMS surfaces were $24.5^{\circ} \mathrm{C} \pm 1{ }^{\circ} \mathrm{C}$ and $25 \pm 2 \%$, respectively. All evaporation experiments were repeated six or more times to ensure the reproducibility. The contact angle hysteresis of pure water droplet on micropatterned PDMS surfaces was measured three or more times for every case following the procedures suggested by Huhtamäki et al. [44] at temperature of $24.5^{\circ} \mathrm{C} \pm 1^{\circ} \mathrm{C}$ and relative humidity of $25 \pm 2 \%$. Contact angle hysteresis of mixture droplets on micro-patterned PDMS surfaces was not measured due to the strong volatility of ethanol.

\section{Results and discussion}

\subsection{Wettability of micro-patterned PDMS surfaces}

Table 1 lists the apparent contact angles [45] of mixture droplets on pillar-arrayed PDMS surfaces. Values of surface roughness $R_{f}=1+\frac{\pi d h}{(d+w)^{2}}$, solid-liquid area fraction $f=\frac{\pi d^{2}}{4(d+w)^{2}}$ and liquid-vapor area fraction $(1-f)$ for patterned PDMS are listed in Table S1. To the best of our knowledge, the apparent contact angle of liquid droplet on rough surface mainly follows four models: (a) Wenzel model [46]: $\cos \theta_{\mathrm{W}}=R_{f} \cos \theta_{\mathrm{Y}}$, where $\theta_{\mathrm{Y}}$ is the intrinsic contact angle of a liquid droplet on an ideal solid surface; (b) Cassie-Baxter model [47]: $\cos \theta_{\mathrm{C}}=f \cos \theta_{\mathrm{Y}}-(1-f)$; (c) mixed model [23]: $\cos \theta_{\mathrm{M}}=\chi \cos \theta_{\mathrm{W}}+(1-\chi) \cos \theta_{\mathrm{C}}$, where $\chi$ is the fraction of the apparent surface which has transitioned to the Wenzel mode and the remaining surface is still Cassie-Baxter-wetted; (d) the triple contact line pinning model [48]. It is already well-known that 

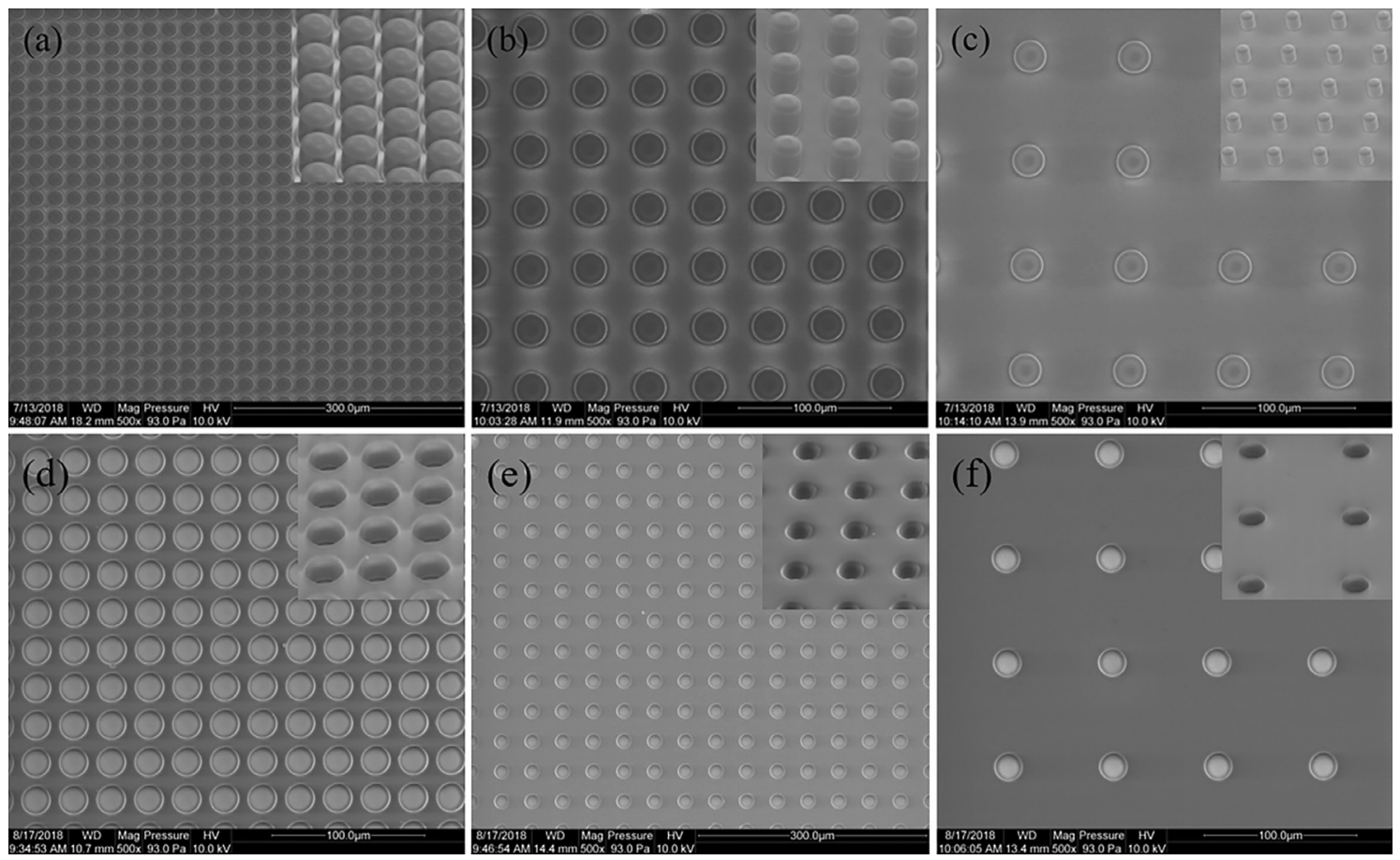

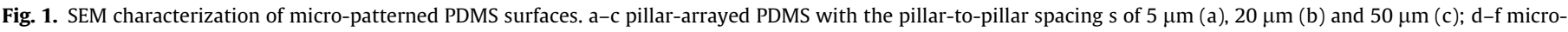
holed PDMS with the hole-to-hole spacing of $5 \mu \mathrm{m}$ (d), $20 \mu \mathrm{m}$ (e) and $50 \mu \mathrm{m}$ (f).

Table 1

Apparent contact angles of mixture droplet on pillar-arrayed PDMS surfaces.

\begin{tabular}{llll}
\hline$C_{\text {ethanol }}$ & $w=5 \mu \mathrm{m}$ & $w=20 \mu \mathrm{m}$ & $w=50 \mu \mathrm{m}$ \\
\hline $0 \%$ & $142^{\circ} \pm 2^{\circ}$ & $147^{\circ} \pm 2^{\circ}$ & $149^{\circ} \pm 2^{\circ}$ \\
$25 \%$ & $136^{\circ} \pm 2^{\circ}$ & $145^{\circ} \pm 2^{\circ}$ & $148^{\circ} \pm 2^{\circ}$ \\
$50 \%$ & $125^{\circ} \pm 2^{\circ}$ & $106^{\circ} \pm 2^{\circ}$ & $93^{\circ} \pm 2^{\circ}$ \\
$75 \%$ & $108^{\circ} \pm 2^{\circ}$ & $83^{\circ} \pm 2^{\circ}$ & $62^{\circ} \pm 2^{\circ}$ \\
\hline
\end{tabular}

the pure Cassie and Wenzel wetting regimes are rare in occurrence, and actually the so-called "mixed wetting state" usually takes place [49-51]. Moreover, Bormashenko et al. [52] elegantly elucidated the physical mechanisms of the wetting transition from Cassie to Wenzel and addressed the energy necessary for de-pinning of the contact line. Indeed, the wetting state should be analyzed from a microscopic view as shown in Refs. [51-53]. For simplification, in this work we used the three models $(a-c)$ to analyze the surface wettability. The apparent contact angles of mixture droplet with initial ethanol concentration of $0 \%, 25 \%, 50 \%$ and $75 \%$ on common PDMS surface at temperature of $26.5^{\circ} \mathrm{C} \pm 1{ }^{\circ} \mathrm{C}$ and relative humidity of $52 \pm 2 \%$ are $116^{\circ} \pm 2^{\circ}, 87^{\circ} \pm 2^{\circ}, 70^{\circ} \pm 2^{\circ}$ and $58^{\circ} \pm 2^{\circ}$, respectively. Substituting these values into the Wenzel model and Cassie-Baxter model, we obtained the corresponding contact angles, as shown in Tables S2 and S3. It was found that the apparent contact angles of mixture droplets on pillar-arrayed surface with the pillar-to-pillar spacing of $5 \mu \mathrm{m}$ are all larger than those determined by Cassie-Baxter model, indicating that there is no liquid penetration into the gap and the surface is Cassie-Baxterwetted. When the spacing increases to $20 \mu \mathrm{m}$ or $50 \mu \mathrm{m}$, the apparent contact angles of mixture droplets on the pillar-arrayed surfaces are all larger than those determined by Wenzel model, while they are all smaller than the values using Cassie-Baxter model. Therefore, the mixed model was used and the values of $\chi$ were calculated and listed in Table S4. The results show that for pure water droplet, the value of $\chi$ for the pillar-to-pillar spacing with $20 \mu \mathrm{m}$ is larger than that with $50 \mu \mathrm{m}$ and there is almost no penetration of liquid into the gap for both cases when the etha- nol concentration is $25 \%$. As the ethanol concentration increases to $50 \%$ and $75 \%$, liquid penetrates the gap and the fraction of penetration increases with the increase of the pillar-to-pillar spacing.

Table 2 shows the apparent contact angles of mixture droplets on micro-holed PDMS surfaces. Values of solid-liquid area fraction $f^{\prime}=1-\frac{\pi d^{2}}{4(d+w)^{2}}$ and liquid-vapor area fraction $\left(1-f^{\prime}\right)$ for patterned PDMS are listed in Table S5. The apparent contact angles of mixture droplet with initial ethanol concentration of $0 \%, 25 \%, 50 \%$ and $75 \%$ on common PDMS surface at temperature of $24.5^{\circ} \mathrm{C} \pm 1{ }^{\circ} \mathrm{C}$ and relative humidity of $25 \pm 2 \%$ are $107^{\circ} \pm 2^{\circ}, 87^{\circ} \pm 2^{\circ}, 61^{\circ} \pm 2^{\circ}$ and $57^{\circ} \pm 2^{\circ}$, respectively. Substituting these values into Cassie-Baxter model, the theoretical contact angles of mixture droplets on micro-holed PDMS surfaces were calculated and listed in Table S6. Comparison between the Cassie contact angles and apparent contact angles demonstrates that all of the micro-holed PDMS surfaces are Cassie-Baxter-wetted.

Table 3 shows the advancing and receding contact angles of pure water droplets on both pillar-arrayed and micro-holed PDMS surfaces. On the pillar-arrayed PDMS surfaces, both the advancing and receding contact angles increase with the increase of the pillar-to-pillar spacing. On the micro-holed PDMS surfaces, both the advancing and receding contact angles decrease with the increase of the hole-to-hole spacing.

\subsection{Evaporation of mixture droplets on micro-patterned PDMS surfaces}

It is demonstrated that the contact line of a pure water droplet is pinned at first on common PDMS surface due to contact angle

Table 2

Apparent contact angles of mixture droplet on micro-holed PDMS surfaces.

\begin{tabular}{llll}
\hline$C_{\text {ethanol }}$ & $w=5 \mu \mathrm{m}$ & $w=20 \mu \mathrm{m}$ & $w=50 \mu \mathrm{m}$ \\
\hline $0 \%$ & $138^{\circ} \pm 2^{\circ}$ & $123^{\circ} \pm 2^{\circ}$ & $116^{\circ} \pm 2^{\circ}$ \\
$25 \%$ & $116^{\circ} \pm 2^{\circ}$ & $97^{\circ} \pm 2^{\circ}$ & $91^{\circ} \pm 2^{\circ}$ \\
$50 \%$ & $111^{\circ} \pm 2^{\circ}$ & $83^{\circ} \pm 2^{\circ}$ & $70^{\circ} \pm 2^{\circ}$ \\
$75 \%$ & $104^{\circ} \pm 2^{\circ}$ & $76^{\circ} \pm 2^{\circ}$ & $68^{\circ} \pm 2^{\circ}$ \\
\hline
\end{tabular}


Table 3

Advancing and receding contact angles of water droplets on micro-patterned PDMS surfaces.

\begin{tabular}{lllll}
\hline Patterned PDMS & $w(\mu \mathrm{m})$ & $\theta_{\mathrm{a}}$ & $\theta_{\mathrm{r}}$ & $\theta_{\mathrm{r}, \text { evap }}$ \\
\hline Pillar-arrayed & 5 & $161^{\circ} \pm 2^{\circ}$ & $110^{\circ} \pm 2^{\circ}$ & $111^{\circ} \pm 2^{\circ}$ \\
& 20 & $171^{\circ} \pm 2^{\circ}$ & $129^{\circ} \pm 2^{\circ}$ & $134^{\circ} \pm 2^{\circ}$ \\
& 50 & $173^{\circ} \pm 2^{\circ}$ & $147^{\circ} \pm 2^{\circ}$ & $145^{\circ} \pm 2^{\circ}$ \\
Micro-holed & 5 & $146^{\circ} \pm 2^{\circ}$ & $96^{\circ} \pm 2^{\circ}$ & $93^{\circ} \pm 2^{\circ}$ \\
& 20 & $132^{\circ} \pm 2^{\circ}$ & $88^{\circ} \pm 2^{\circ}$ & $85^{\circ} \pm 2^{\circ}$ \\
& 50 & $127^{\circ} \pm 2^{\circ}$ & $84^{\circ} \pm 2^{\circ}$ & $85^{\circ} \pm 2^{\circ}$ \\
\hline
\end{tabular}

hysteresis, and depinning of the contact line of ethanol/water mixture droplet is observed on the surface throughout the whole evaporation process $[10,43,54]$. However, it is not the case for ethanol/ water droplets on the pillar-arrayed PDMS surfaces. Figs. 2 and 3 show the evolution of contact radius and contact angle of evaporating mixture droplets on the pillar-arrayed PDMS surfaces, respectively. It can be seen that all droplets first experience CCR mode and the duration of CCR stage decreases with the increase of the pillar-to-pillar spacing. The difference in evaporation characteristics between common and the pillar-arrayed PDMS surfaces may be attributed to the strong adhesion [55] between each of the pillar-arrayed PDMS surfaces and the droplet. As the pillar-topillar spacing increases, the adhesion becomes less and then a shorter CCR stage is found. Moreover, the receding contact angles of pure water droplets on the pillar-arrayed PDMS surfaces can also be determined from Figs. 2 and 3 and are listed in Table 3, indicating that the measured receding contact angles using an NE31 type needle are nearly equal to those determined by evaporation, which is consistent with Ref. [56].
When the contact lines begin to recede, the contact angle nearly keeps constant for a long period for $0 \%, 25 \%$ and $50 \%$ ethanol/water droplets on the pillar-arrayed PDMS surfaces with the pillar-topillar spacing of 20 or $50 \mu \mathrm{m}$. For mixture droplets with the initial ethanol concentration of $75 \%$ on the pillar-arrayed PDMS surfaces with the pillar-to-pillar spacing of 20 or $50 \mu \mathrm{m}$, the contact angle begins to increase at the end of the first stage-CCR stage and increases to a maximum. This phenomenon is similar to that observed by Tadmor $[57,58]$. Due to the high volatility of ethanol, there will be a greater increase in liquid-vapor interfacial tension as well as a slight increase in solid-liquid interfacial tension (as shown in Fig. $3 \mathrm{~d}$, at the end of CCR stage, the contact angle is much less than $90^{\circ}$ ), resulting in the retraction of the contact line and the increase of the contact angle. As the contact angle reaches the maximum, the contact angle begins to decrease with decreasing contact radius for the pillar-arrayed PDMS surface with the pillar-topillar spacing of $20 \mu \mathrm{m}$, while the contact radius keeps unchanged and the contact angle decreases continuously for the pillar-arrayed surface with the pillar-to-pillar spacing of $50 \mu \mathrm{m}$.

Figs. 4 and 5 show the evolution of contact radius and contact angle of evaporating mixture droplets on micro-holed PDMS surfaces, respectively. For pure water droplets, CCR mode is observed at first on all micro-holed PDMS surfaces and the duration of CCR stage decreases with the increase of the hole-to-hole spacing because the increase of the spacing will weaken the adhesion between the patterned surface and the droplet. The receding contact angles determined by evaporation are also compared with those using the needle method as listed in Table 3, indicating the receding contact angle on the micro-holed PDMS surfaces can also be measured from evaporation. For $25 \%$ ethanol/water droplet, the contact angle keeps nearly unchanged for a relatively long time
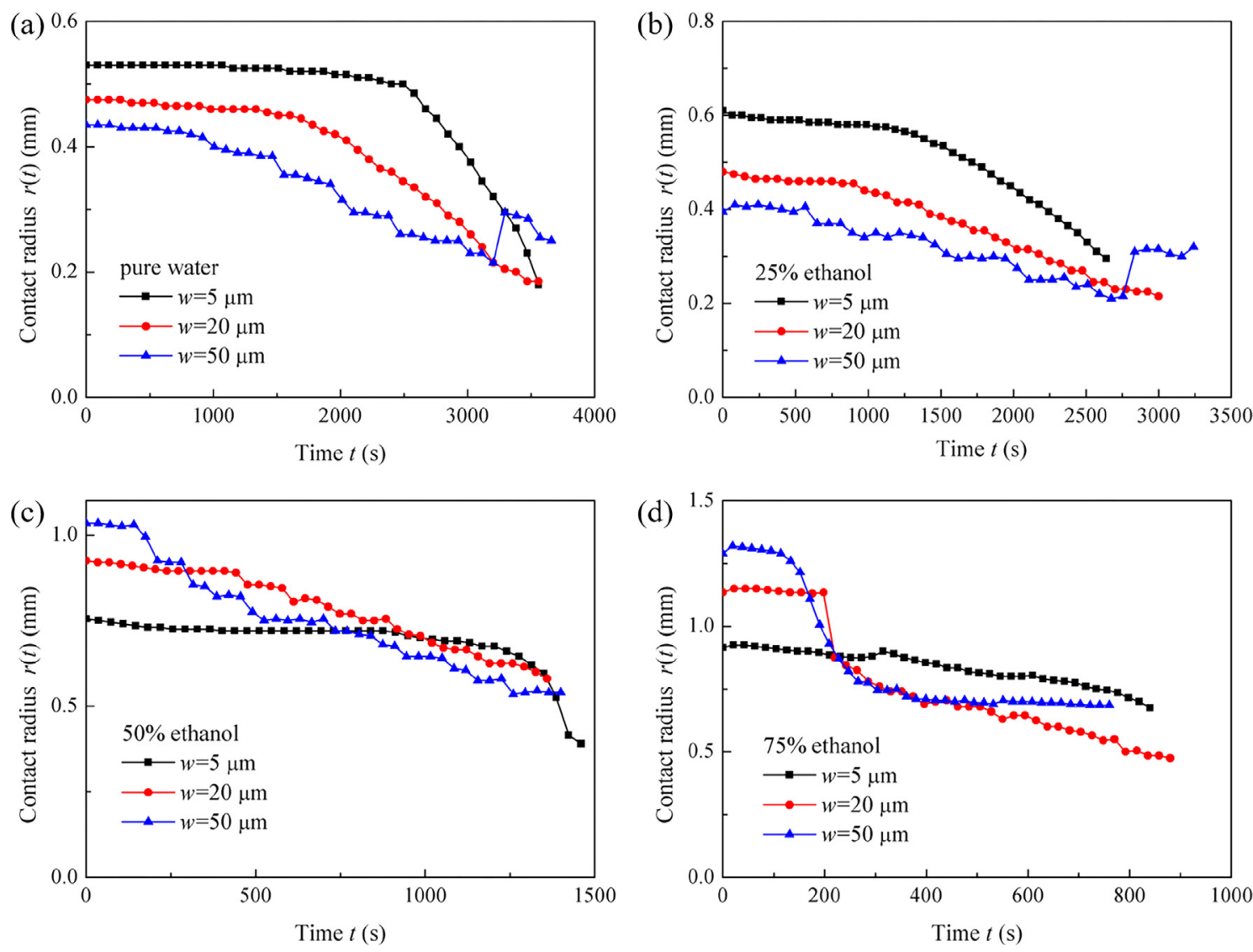

Fig. 2. Contact radius of evaporating mixture droplets on pillar-arrayed PDMS surfaces. a pure water, b $25 \%$ ethanol, c $50 \%$ ethanol, d $75 \%$ ethanol. 

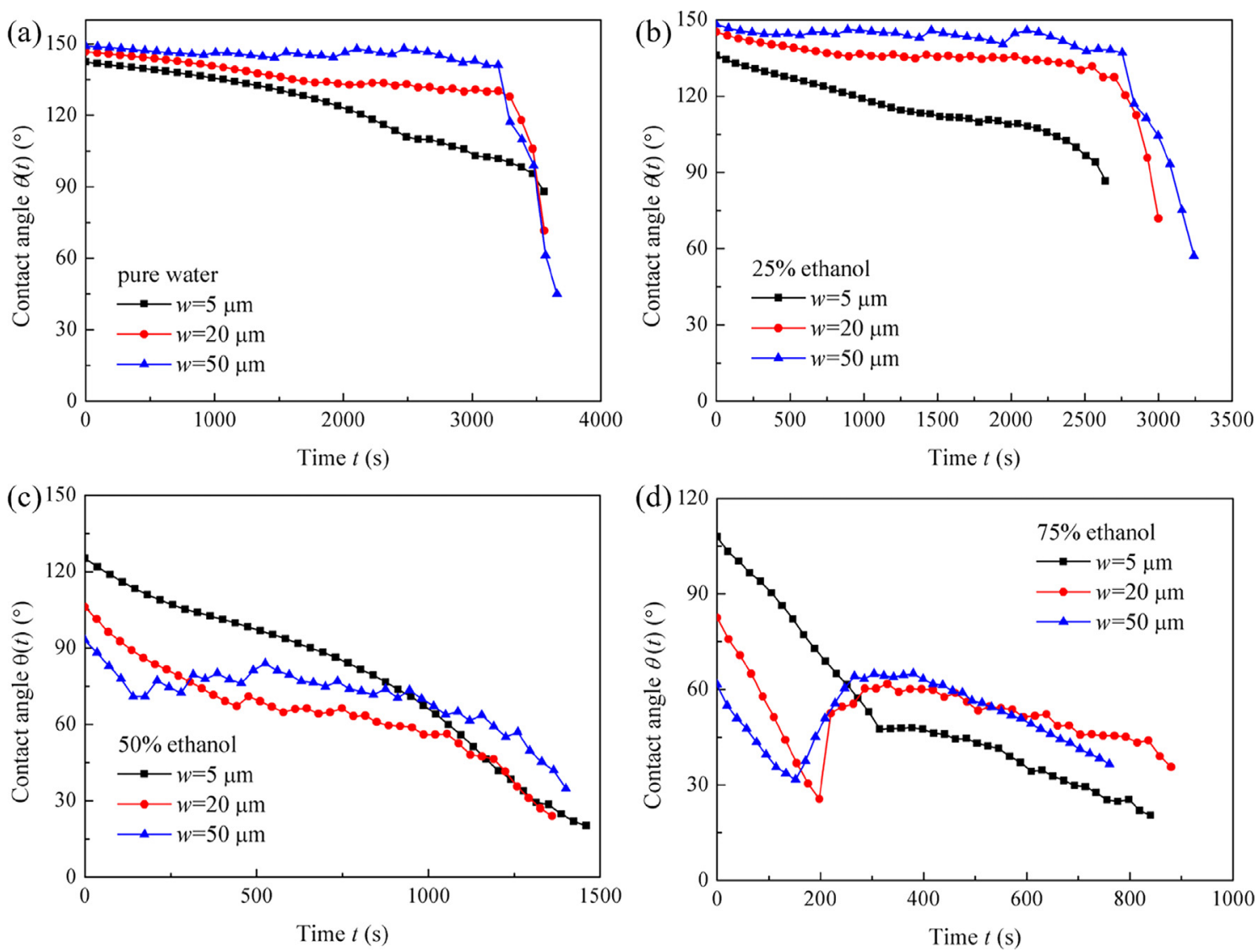

Fig. 3. Contact angle of evaporating mixture droplets on pillar-arrayed PDMS surfaces. a pure water, b $25 \%$ ethanol, c $50 \%$ ethanol, d $75 \%$ ethanol.
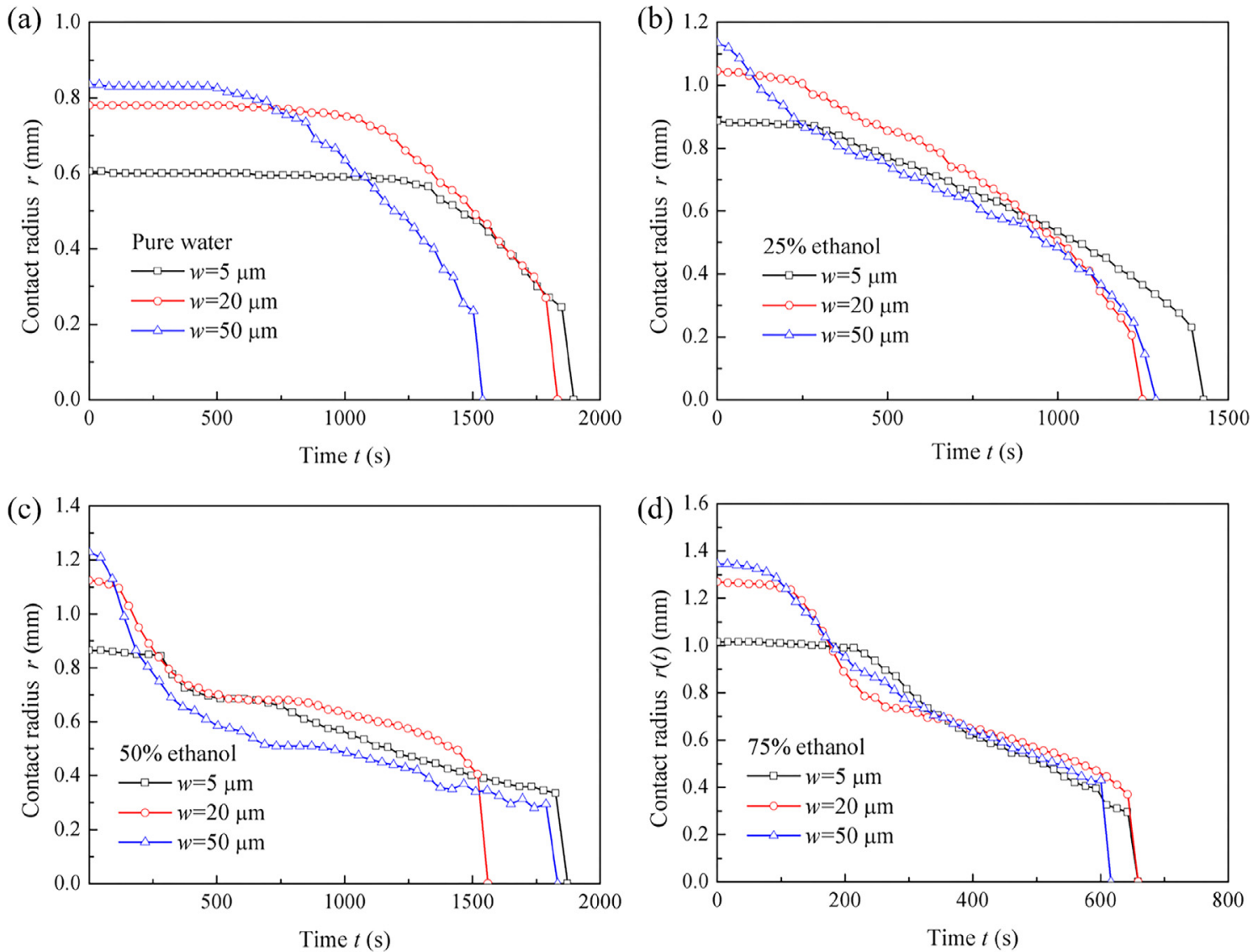

Fig. 4. Contact radius of evaporating mixture droplets on micro-holed PDMS surfaces. a pure water, b $25 \%$ ethanol, c $50 \%$ ethanol, d $75 \%$ ethanol. 

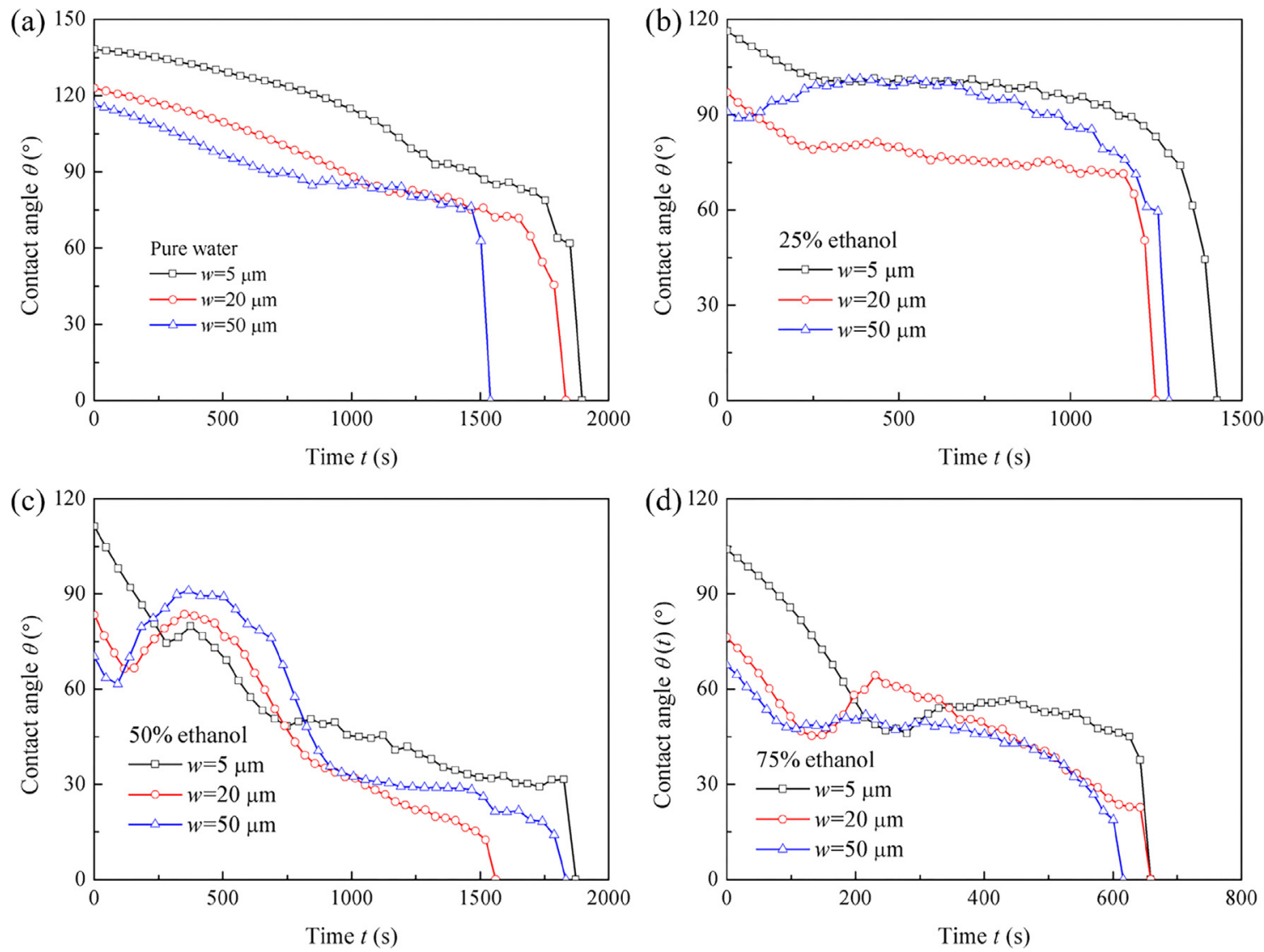

Fig. 5. Contact angle of evaporating mixture droplets on micro-holed PDMS surfaces. a pure water, b $25 \%$ ethanol, c 50\% ethanol, d $75 \%$ ethanol.
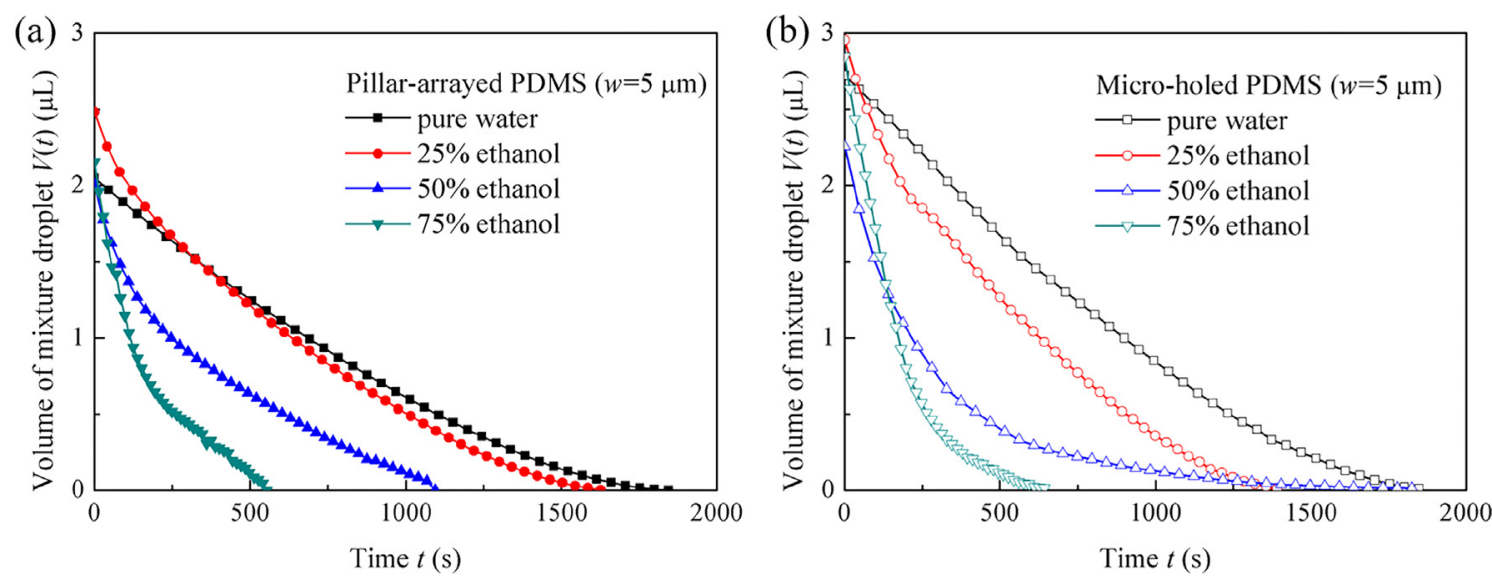

Fig. 6. Volume of evaporating mixture droplets on micro-patterned PDMS surfaces. a pillar-arrayed $(w=5 \mu \mathrm{m}), \mathrm{b}$ micro-holed $(w=5 \mu \mathrm{m})$.

from the end of the CCR stage on the micro-holed surfaces with the hole-to-hole spacing of 5 or $20 \mu \mathrm{m}$, and it is not the case for the surface with the hole-to-hole spacing of $50 \mu \mathrm{m}$. On that surface, the contact angle has a slight decrease at first and then begins to increase. When it increases to the maximum, the contact angle keeps nearly unchanged for a period and finally it continuously decreases. The contact radius decreases throughout the whole process. For $50 \%$ and $75 \%$ ethanol/water droplets, there are three or four characteristic stages of contact angle. At first, the contact angle decreases with time, then it continuously increases until it reached to the maximum and keeps nearly unchanged for a period (sometimes no CCA stage is observed) and finally it decreases con- tinuously. The stage at which the contact angle continues to increase can also be explained by using Tadmor's theory $[57,58]$.

Fig. 6 shows the volume evolution of evaporating mixture droplet on the micro-patterned PDMS surfaces with the pillar-to-pillar or hole-to-hole spacing of $5 \mu \mathrm{m}$. For the pillar-arrayed PDMS surface, the volume curve of a $25 \%$ ethanol/water mixture droplet slightly deviates from that of a pure water droplet. On the microholed PDMS surface, a greater deviation is observed. For $50 \%$ and $75 \%$ ethanol/water droplet, two different stages are observed. In the first stage, volume decreases sharply due to the high volatility of ethanol. In the second stage, volume decreases slowly because evaporation of water dominates. 

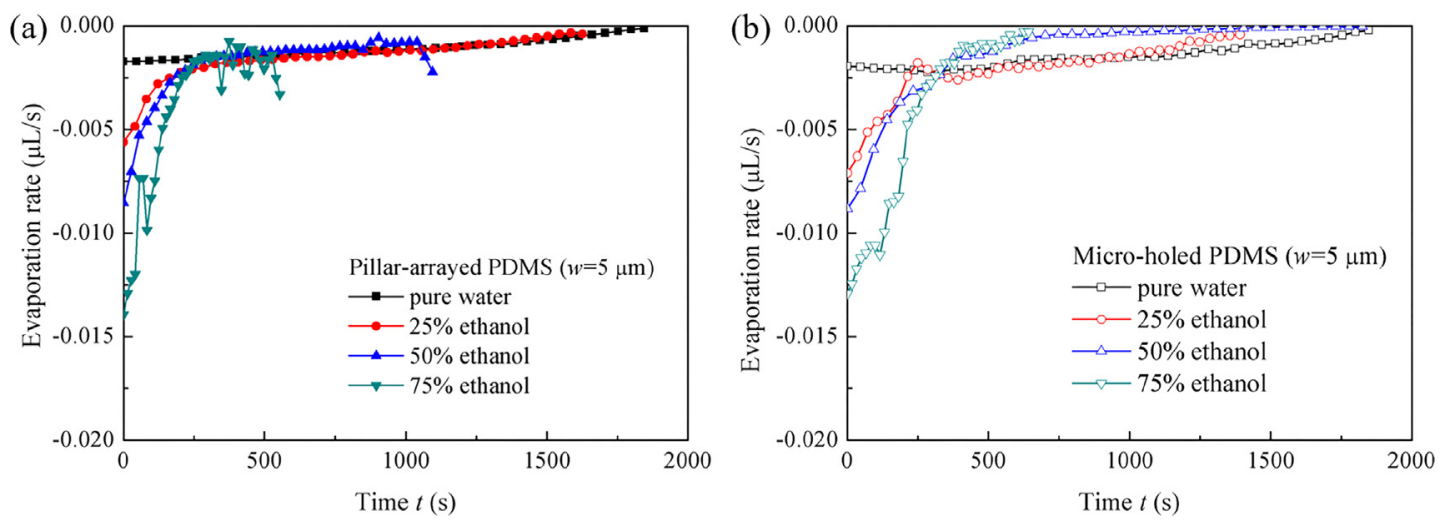

Fig. 7. Evaporation rate of mixture droplets on micro-patterned PDMS surfaces. a pillar-arrayed $(w=5 \mu \mathrm{m})$, $\mathrm{b}$ micro-holed $(w=5 \mu \mathrm{m})$.
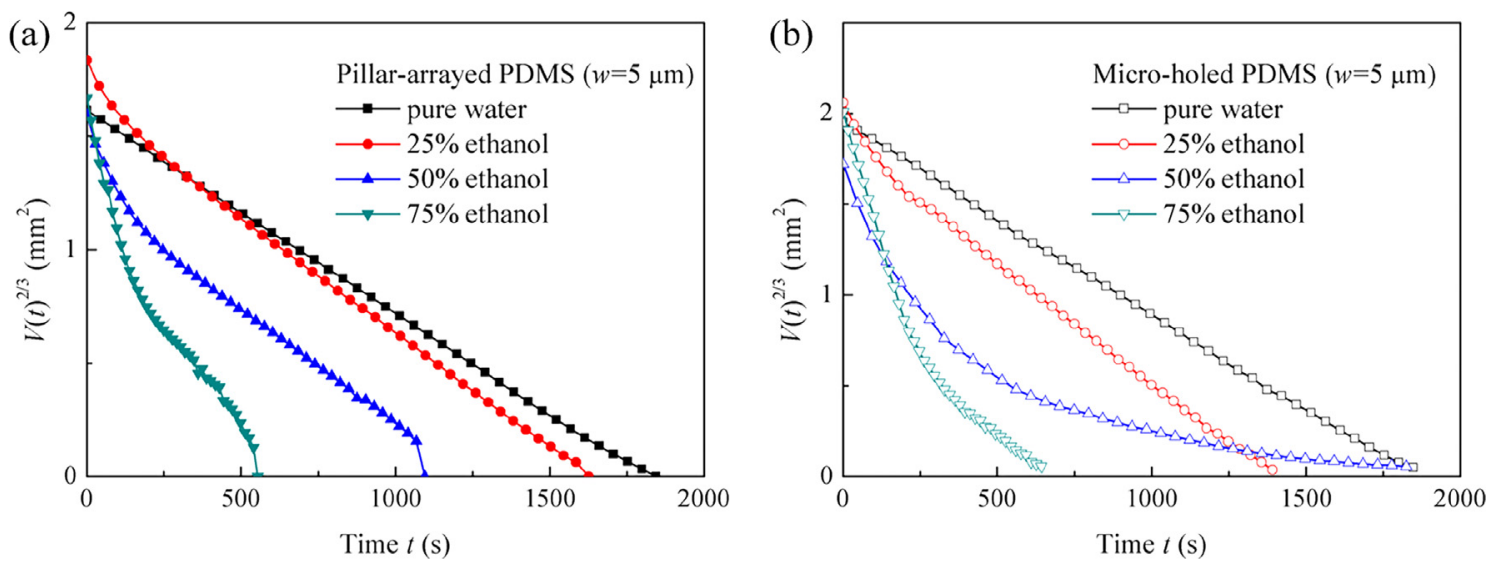

Fig. 8. Two-third power of mixture droplet volume on micro-patterned PDMS surfaces. a pillar-arrayed $(w=5 \mu \mathrm{m})$, $\mathrm{b}$ micro-holed $(w=5 \mu \mathrm{m})$.

Fig. 7 shows evaporation rate of mixture droplets on the micropatterned surfaces with the pillar-to-pillar or hole-to-hole spacing of $5 \mu \mathrm{m}$. Evaporation rates of pure water droplets on the pillararrayed and micro-holed PDMS surfaces are of the order of $-0.002 \mu \mathrm{L} / \mathrm{s}$ at first and continuously increase to about 0 . When ethanol is introduced into the droplet, the evaporation rate of mixture droplet decreases with increasing ethanol concentration and becomes equal to that of pure water droplet when enough ethanol has volatilized.

Fig. 8 shows the two-third power of volume of mixture droplets on the micro-patterned PDMS surfaces with the pillar-to-pillar or hole-to-hole spacing of $5 \mu \mathrm{m}$. For pure water droplet, two-third power of volume decreases linearly with time, which is consistent with Ref. [59]. A piecewise bilinear stage is found for ethanol/water mixture droplets. At the first linear stage, evaporation of mixture droplets is mainly attributed to the volatility of ethanol. When ethanol concentration decreases to a low value, the evaporation switches to the second linear stage in which water evaporation dominates.

\section{Conclusion}

In this work, the wettability of micro-patterned PDMS surfaces and evaporation of ethanol/water mixture droplets on the surfaces were studied. Pillar-arrayed PDMS surfaces with the pillar-to-pillar spacing of $5 \mu \mathrm{m}$ is Cassie-Baxter-wetted. When the pillar-to-pillar spacing increases to 20 or $50 \mu \mathrm{m}$, part of the surfaces is CassieBaxter-wetted and the other part Wenzel-wetted. All the microholed PDMS surfaces are Cassie-Baxter-wetted. The advancing and receding contact angles of pure water droplets on the micro- patterned PDMS surfaces both increase with the increase of the pillar-to-pillar spacing, while they decrease with the increase of the hole-to-hole spacing. CCR mode is found in most of the evaporation experiments of ethanol/water mixture droplets, which is attributed to the strong adhesion between the surface and the droplet. The duration of CCR stage increases with the increase of the pillar-to-pillar spacing, while it decreases with the increase of the hole-to-hole spacing. Evolution of droplet volume and its two-third power as well as evaporation rate are discussed. For pure water droplet, the two-third power of droplet volume decreases linearly with time. As ethanol is introduced into water, it decreases sharply at first for a period and then switches to a slow decrease as more and more ethanol has volatilized.

\section{Declaration of Competing Interest}

The authors declared that there is no conflict of interest.

\section{Acknowledgements}

This work was jointly supported by the National Natural Science Foundation of China (Grant Nos. 11572114, 11702299), the Chinese Academy of Sciences Strategic Priority Research Program (Grant No. XDB22040401) and the Opening Fund of Laboratory of Nonlinear Mechanics, Chinese Academy of Sciences. We also thank Prof. Ya-Pu Zhao from Institute of Mechanics, Chinese Academy of Sciences, and Prof. Dong Han and Dr. Jiantao Feng from National Center for Nanoscience and Technology, Chinese Academy of Sciences for experimental support. 


\section{Appendix A. Supplementary material}

Supplementary data to this article can be found online at https://doi.org/10.1016/j.ijheatmasstransfer.2019.118708.

\section{References}

[1] H.Y. Erbil, Evaporation of pure liquid sessile and spherical suspended drops: a review, Adv. Colloid Interf. Sci. 170 (2012) 67-86.

[2] Y.P. Zhao, Physical Mechanics of Surfaces and Interfaces, Science Press, Beijing, 2012.

[3] Y.P. Zhao, Moving contact line problem: advances and perspectives, Theor. Appl. Mech. Lett. 4 (2014) 034002.

[4] P.S. Wasnik, H.E. N'Guessan, R. Tadmor, Controlling arbitrary humidity without convection, J. Colloid Interf. Sci. 455 (2015) 212-219.

[5] R. Tadmor, P.S. Wasnik, H.E. N'guessan, R. Tadmor, M. Tadmor, Inducing arbitrary vapor pressures, and quantifying leakages, AIChE J. 62 (2016) 45484553.

[6] M. Parsa, S. Harmond, K. Sefiane, Mechanisms of pattern formation from dried sessile drops, Adv. Colloid Interf. Sci. 254 (2018) 22-47.

[7] D.Y. Zang, S. Tarafdar, Y.Y. Tarasevich, M.D. Choudhury, T. Dutta, Evaporation of a droplet: from physics to applications, Phys. Rep. 804 (2019) 1-56.

[8] R.G. Picknett, R. Bexon, The evaporation of sessile or pendant drops in still air, J. Colloid Interf. Sci. 61 (2) (1977) 336-350.

[9] S.M. Rowan, M.I. Newton, G. McHale, Evaporation of microdroplets and the wetting of solid surfaces, J. Phys. Chem. 99 (35) (1995) 13268-13271.

[10] Y.S. Yu, Z.Q. Wang, Y.P. Zhao, Experimental and theoretical investigations of evaporation of sessile water droplet on hydrophobic surfaces, J. Colloid Interf. Sci. 365 (2012) 254-259.

[11] X.M. Chen, R.Y. Ma, J.T. Li, C.L. Hao, W. Guo, B.L. Luk, S.C. Li, S.H. Yao, Z.K. Wang, Evaporation of droplets on superhydrophobic surfaces: surface roughness and small droplet size effects, Phys. Rev. Lett. 109 (11) (2012) 116101.

[12] K. Sefiane, L. Tadrist, M. Douglas, Experimental study of evaporating waterethanol mixture sessile drop: influence of concentration, Int. J. Heat Mass Transf. 46 (23) (2003) 4527-4534.

[13] P. Innocenzi, L. Malfatti, S. Costacurta, T. Kidchob, M. Piccinini, A. Marcelli, Evaporation of ethanol and ethanol-water mixtures studied by time-resolved infrared spectroscopy, J. Phys. Chem. A 112 (29) (2008) 6512-6516.

[14] C.J. Liu, E. Bonaccurso, H.J. Butt, Evaporation of sessile water/ethanol drops in a controlled environment, Phys. Chem. Chem. Phys. 10 (47) (2008) 7150-7157.

[15] Z. Wang, X.F. Peng, A.S. Mujumdar, A. Su, D.J. Lee, Evaporation of ethanol-water mixture drop on horizontal substrate, Dry. Technol. 26 (6) (2008) 806-810.

[16] L.X. Shi, P. Shen, D. Zhang, Q.L. Lin, Q.C. Jiang, Wetting and evaporation behaviors of water-ethanol sessile drops on PTFE surfaces, Surf. Interf. Anal. 41 (12-13) (2009) 951-955.

[17] C.H. Ooi, E. Bormashenko, A.V. Nguyen, G.M. Evans, D.V. Dao, N.T. Nguyen, Evaporation of ethanol-water binary mixture sessile liquid marbles, Langmuir 32 (24) (2016) 6097-6104.

[18] S.M. Rowan, M.I. Newton, F.W. Driewer, G. McHale, Evaporation of microdroplets of azeotropic liquids, J. Phys. Chem. B 104 (34) (2000) 8217-8220.

[19] M. Rusdi, Y. Moroi, H. Nakahara, O. Shibata, Evaporation from water-ethylene glycol liquid mixture, Langmuir 21 (16) (2005) 7308-7310.

[20] K. Sefiane, S. David, M.E.R. Shanahan, Wetting and evaporation of binary mixture drops, J. Phys. Chem. B 112 (36) (2008) 11317-11323.

[21] C. Diddens, J.G.M. Kuerten, C.W.M. van der Geld, H.M.A. Wijshoff, Modeling the evaporation of sessile multi-component droplets, J. Colloid Interf. Sci. 487 (2017) 426-436.

[22] J.R.E. Christy, Y. Hamamoto, K. Sefiane, Flow transition within an evaporating binary mixture sessile drop, Phys. Rev. Lett. 106 (20) (2011) 205701.

[23] Q.S. Zheng, Y. Yu, Z.H. Zhao, Effects of hydraulic pressure on the stability and transition of wetting modes of superhydrophobic surfaces, Langmuir 21 (26) (2005) 12207-12212.

[24] Q.Z. Yuan, Y.P. Zhao, Wetting on flexible hydrophilic pillar-arrays, Sci. Rep. 3 (2013) 1944

[25] P.T. Wang, J.W. Su, M.Y. Shen, M. Ruths, H.W. Sun, Detection of liquid penetration of a micropillar surface using the quartz crystal microbalance, Langmuir 33 (2) (2017) 638-644.

[26] H.P. Wu, K. Zhu, B.B. Cao, Z. Zhang, B.B. Wu, L.H. Liang, G.Z. Chai, A.P. Liu, Smart design of wettability-patterned gradients on substrate-independent coated surfaces to control unidirectional spreading of droplets, Soft Matter 13 (16) (2017) 2995-3002.

[27] H.P. Wu, Z. Yang, B.B. Cao, Z. Zhang, K. Zhu, S.F. Jiang, G.Z. Chai, Wetting and dewetting transitions on submerged superhydrophobic surfaces with hierarchical structures, Langmuir 33 (1) (2017) 407-416.
[28] Y.L. Xiang, S.L. Huang, P.Y. Lv, Y.H. Xue, Q. Su, H.L. Duan, Ultimate stable underwater superhydrophobic state, Phys. Rev. Lett. 119 (13) (2017) 134501.

[29] A. Lafuma, D. Quéré, Superhydrophobic states, Nature Mater. 2 (7) (2003) 457 460.

[30] Q.Z. Yuan, Y.P. Zhao, Multiscale dynamic wetting of a droplet on a lyophilic pillar-arrayed surface, J. Fluid Mech. 716 (2013) 171-188.

[31] Q.Z. Yuan, X.F. Huang, Y.P. Zhao, Dynamic spreading on pillar-arrayed surfaces: viscous resistance versus molecular friction, Phys. Fluids 26 (9) (2014) 092104.

[32] E.H. Chen, Q.Z. Yuan, Y.P. Zhao, Topography-induced symmetry of droplets on quasi-periodically patterned surfaces, Soft Matter 14 (30) (2018) 6198-6205.

[33] N. Anantharaju, M. Panchagnula, S. Neti, Evaporating drops on patterned surfaces: transition from pinned to moving triple line, J. Colloid Interf. Sci. 337 (1) (2009) 176-182.

[34] C. Luo, M.M. Xiang, X.C. Liu, Transition from Cassie-Baxter to Wenzel states on micronline-formed PDMS surfaces induced by evaporation or pressing of water droplets, Microfluid Nanofluid 10 (4) (2011) 831-842.

[35] C. Zhang, X.L. Zhu, L.W. Zhou, Morphology tunable pinning force and evaporation modes of water droplets on PDMS spherical cap micron-arrays, Chem. Phys. Lett. 508 (1-3) (2011) 134-138.

[36] Y.C. Chuang, C.K. Chu, S.Y. Lin, L.J. Chen, Evaporation of water droplets on soft patterned surfaces, Soft Matter 10 (19) (2014) 3394-3403.

[37] G. McHale, S. Aqil, N.J. Shirteliffe, M.I. Newton, H.Y. Erbil, Analysis of droplet evaporation on a superhydrophobic surface, Langmuir 21 (24) (2005) 1105311060.

[38] W. Xu, R. Leeladhar, Y.T. Kang, C.H. Choi, Evaporation kinetics of sessile water droplets on micropatterned superhydrophobic surfaces, Langmuir 29 (20) (2013) 6032-6041.

[39] R. Chen, L. Jiao, X. Zhu, Q. Liao, D.D. Ye, B. Zhang, W. Li, Y.P. Lei, D.L. Li, Cassieto-Wenzel transition of droplet on the superhydrophobic surface caused by light induced evaporation, Appl. Therm. Eng. 144 (2018) 945-959.

[40] F.C. Wang, H.A. Wu, Pinning and depinning mechanism of the contact line during evaporation of nano-droplets sessile on textured surfaces, Soft Matter 9 (23) (2013) 5703-5709.

[41] F.C. Wang, H.A. Wu, Molecular origin of contact line stick-slip motion during droplet evaporation, Sci. Rep. 5 (2015) 17521.

[42] M.H. He, D. Liao, H.H. Qiu, Multicomponent droplet evaporation on chemical micro-patterned surfaces, Sci. Rep. 7 (2017) 41897.

[43] Y.S. Yu, L. Sun, X.F. Huang, J.Z. Zhou, Evaporation of ethanol/water mixture droplet on a pillar-like PDMS surface, Colloid Surf. A 574 (2019) 215-220.

[44] T. Huhtamäki, X.L. Tian, J.T. Korhonen, R.H.A. Ras, Surface-wetting characterization using contact-angle measurements, Nat. Protoc. 13 (7) (2018) 1521-1538.

[45] A. Marmur, C.D. Volpe, S. Siboni, A. Amirfazli, J.W. Drelich, Contact angles and wettability: towards common and accurate terminology, Surf. Innov. 5 (1) (2017) 3-8.

[46] R.N. Wenzel, Resistance of solid surfaces to wetting by water, Ind. Eng. Chem. 28 (8) (1936) 988-994.

[47] A.B.D. Cassie, S. Baxter, Wettability of porous surfaces, Trans. Faraday Soc. 40 (1944) 546-551.

[48] J.L. Liu, Y. Mei, R. Xia, A new wetting mechanism based upon triple contact line pinning, Langmuir 27 (1) (2011) 196-200.

[49] M. Miwa, A. Nakajima, A. Fujishima, K. Hashimoto, T. Watanabe, Effects of the surface roughness on sliding angles of water droplets on superhydrophobic surfaces, Langmuir 16 (13) (2000) 5754-5760.

[50] A. Marmur, Wetting on hydrophobic rough surfaces: to be heterogeneous or not to be?, Langmuir 19 (20) (2003) 8343-8348

[51] E. Bormashenko, Progress in understanding of wetting transitions on rough surfaces, Adv. Colloid Interf. Sci. 222 (2015) 92-103.

[52] E. Bormashenko, A. Musin, G. Whyman, M. Zinigrad, Wetting transitions and depinning of the triple line, Langmuir 28 (7) (2012) 3460-3464.

[53] J.L. Liu, J. Sun, Y. Mei, Biomimetic mechanics behaviors of the strider leg vertically pressing water, Appl. Phys. Lett. 104 (23) (2014) 231607.

[54] Y.S. Yu, Z.Q. Wang, Y.P. Zhao, Experimental study of evaporation of sessile water droplet on PDMS surfaces, Acta Mech. Sin. 29 (6) (2013) 799-805.

[55] R. Tadmor, R. Das, S. Gulec, J. Liu, H.E. N'guessan, M. Shah, P.S. Wasnik, S.B. Yadav, Solid-liquid work of adhesion, Langmuir 33 (15) (2017) 3594-3600.

[56] H.Y. Erbil, G. McHale, S.M. Rowan, M.I. Newton, Determination of the receding contact angle of sessile drops on polymer surfaces by evaporation, Langmuir 15 (21) (1999) 7378-7385.

[57] R. Tadmor, Drops that pull themselves up, Surf. Sci. 628 (2014) 17-20.

[58] R. Tadmor, A. Baksi, S. Gulec, S. Jahav, H.E. N'guessan, K. Sen, V. Somasi, M Tadmor, P. Wasnik, S.B. Yadav, Drops that change their mind: spontaneous reversal from spreading to retraction, Langmuir (2019), https://doi.org/ 10.1021/acs.langmuir.9b02592.

[59] T.A.H. Nguyen, A.V. Nguyen, Transient volume of evaporating sessile droplets: $2 / 3,1 / 1$, or another power law?, Langmuir 30 (22) (2014) 6544-6547 